\title{
Agent-based Simulation Study on Mode of Electric Vehicle Refueling Station
}

\author{
Yingqi $\mathrm{Ni}^{1,}$ a , Peipei Liu'b ${ }^{\mathrm{b}}$, Jiangjiang Zhao ${ }^{3, \mathrm{c}}$ and Tieju Ma ${ }^{4, \mathrm{~d}}$ \\ 1,2,3,4 Business School, East China University of Science and Technology, \\ Shanghai, 200237, China \\ aniyingqi@163.com, ${ }^{b}$ peipei@163.com, cjiangjz@126.com, ditja@@ecust.edu.cn
}

\begin{abstract}
Keywords: Electric vehicle; Battery swap; Fast charging; Agent-based modeling.
Abstract. With the deterioration of air pollution, global warming, people pay more attention to electric vehicles(EV). For the relationship between the diffusion of EVs and establishment of refueling stations, it is inter-constraining, and inter-stimulating. Based on agent-based model, we compare the effects of diffusion of EVs respectively through the simulation on fast charging and battery changing mode, and put forward policy proposals on the effective way of subsidy. Simulation results has shown fast charging station is more favorable toward the diffusion of electric vehicles.
\end{abstract}

\section{Introduction}

Due to air pollution, electric vehicles(EV) has caught people's concerns[1]. Promoting uptake of EVs needs the development of infrastructure, because relation between EV uptake and their infrastructure is interacting, as refueling convenience could enhance EVs uptake, and increased EV market share will stimulate energy suppliers to build more refueling stations[2]. Moreover, there are 3 available mode of refueling, slow charging, fast charging(FC) and battery swap(BS)[3]. For public EV stations, FC and BS are mainly adopted. Fast charging use large direct currents to fully recharge a electric car in 20 minutes to 2 hours, while battery swap refuels vehicles through direct replacement of fully charged battery in 5 minutes. Detail cost description of FC and BS could be found in section 3 and 4 .

For our methodology, Agent Based Modeling(ABM) is a bottom-to-up modeling method. Agents are adaptable, pro-activeness and could change their behaviors through continuous learning coming from interaction with surroundings[4]. Building a complex adaptive system, agents are taken as system's basic units by giving them specific rules separately, and setting rules for interaction among multiple agents. Now, scholars have made some achievement in the study of electric vehicles on the basis of ABM. For example, they have studied EV's market penetration[5] and EV charging network distribution[6].

Our paper tend to study the impact of fast charging and battery swap mode to uptake of EV on the basis of an ABM model. Moreover, we will take Shanghai as an example to simulate which mode perform better to improve EV uptake.

\section{Model Introduction}

The diffusing model we used is for analyzing interacting relationship between EV drivers and refueling stations on the basis of ABM[7], which includes two types of agents: EV-driver agents and refueling-station-operator agents. Each EV-driver agent will consider changing her car every month with two option: a gasoline car or an EV based on evaluation utility, according to Eq. 1.

$$
U=\text { fixedBenefit }+ \text { totalVariableBenefit }+ \text { socialValue }- \text { worryfactor }
$$

FixedBenefit is earning as buying a gasoline car. TotalVariableBenefit means energy benefit. SocialValue is benefits from public opinions due to purchasing environmental-friendly cars. WorryFactor is driver's range anxiety. If $\mathrm{U}>0$, consumer will choose $\mathrm{EV}$. If not, driver will choose gasoline cars.

Moreover, model assumes each road intersection could be a potential refueling station. Every month, each refueling-station-operator agent will evaluate station's performance to consider whether to run or close based on refueling station utility according to Eq. 2, and Eq. 3. 
PRICE_OF_ELECTRICITY is average electricity price; totalFlowRate is total number of EVs which have past the node in the last month. TotalBenefit indicates the whole benefit of refueling station. Threshold indicates the minimum level of profitability; basicStationCost indicates stations' construction cost; batteryStationCost indicates battery purchasing cost; operationCost is operational cost; variableBatteryCost indicates stations' electricity costs. When $U>0$, they will build a refueling station. If not, they will close the station.

In the model, there are 8000 drivers. At the beginning of diffusion, 10 refueling stations are served as seeds and $1 \%$ of drivers are EV users.

\section{Simulating Analysis Of Fast Charging Stations}

The cost of a fast charging station is comprised by initial investment cost and operating cost. The detail of cost could be found in Table 1[8].In the simulation, each step represents for one month, so 180 steps means 15 years, which is the same as a life cycle of fast charging station. For the whole simulation, we try to see fast charging stations' influence to the diffusion of electric cars in one life cycle. To reduce randomness, we will take the average number as our simulation result after 10 times of tests in each specific scenario. The simulation result are as follows:

Sensitivity of TLR, Initial Investment, and Labor Cost. To study influence of different technical learning rate(TLR), we compare EVs' ratio when TLR is $0.08,0.10$ and 0.15 ,construction cost, annual labor cost are 4.36 million and 240,000 Yuan. From Fig.1, we could see the higher technical learning rate is, the faster electric vehicles diffusion will be. When TLR reaches 0.15 , EVs could completely replace gasoline cars in less than 10 years.

To see the influence of initial investment cost, we compare the ratio of EVs, when initial investment cost is in 336, 436, 536 million Yuan, TLR is set in 0.1, and annual labor cost is 240,000 Yuan. From Fig. 2, we could see that the higher initial investment cost is, the slower electric vehicles diffusion will be. Moreover, comparing to our estimated actual initial investment cost, 4.36 million, the boost of diffusion caused by 1million cost decrease is much higher than the decline of diffusion caused by 1 million cost increase. If government could provide 1 million initial investment subsidy to the station operators, the effectiveness of influence of fast charging station to the diffusion of electric cars could rapidly rocket to $80 \%$ in the first 4.5 years and completely replace gasoline cars after 12 years.

For labor cost, to see the influence of labor cost to the diffusion of electric cars, we compare the ratio of EVs, when labor cost is set in 15, 20, 25 thousand Yuan per month, and technical learning rate is set in 0.1 ,initial investment cost is 4.36 million Yuan. According to Fig. 3, we could see that the higher labor cost is, the slower electric cars diffusion will be.

Table 1. Cost Of a Fast Charging Station

\begin{tabular}{|c|c|c|}
\hline Cost Type & Specific Contents & Fee(10 thousand Yuan) \\
\hline \multirow{3}{*}{$\begin{array}{l}\text { Initial } \\
\text { investment }\end{array}$} & 10 fast chargers & 200 \\
\hline & Maintain, monitoring facilities, installing & 40 \\
\hline & Distribution facilities, installing & 196 \\
\hline \multicolumn{2}{|c|}{ Total initial investment cost } & 436 \\
\hline \multirow{4}{*}{ Operating cost } & Labor cost & Per year 24.000 \\
\hline & Electricity cost & Per year $0.528 \times$ totalFlowRate \\
\hline & Depreciation cost & Per year 29.212 \\
\hline & Maintenance cost & Per year 8.720 \\
\hline \multicolumn{2}{|c|}{ Total Operating cost } & $\begin{array}{l}\text { Per year } \\
61.932+0.528 \times \text { totalFlowRate }\end{array}$ \\
\hline
\end{tabular}




\section{Simulation Analysis Of Battery Swap Stations}

Table 2. Cost Of a Battery Swap Station

\begin{tabular}{|c|c|c|}
\hline Cost type & Specific Contents & Fee(Thousand Yuan) \\
\hline \multirow[t]{4}{*}{ Initial investment Cost } & 8 automatic and semi-automatic equipment & 4700 \\
\hline & Distribution, maintenance, security facilities & 300 \\
\hline & Total Construction Cost & 5000 \\
\hline & 500 batteries & 5000 \\
\hline \multicolumn{2}{|c|}{ Total initial investment cost } & 10,000 \\
\hline \multirow[t]{5}{*}{ Operating Cost } & Depreciation cost & 335 \\
\hline & Maintenance cost & 100 \\
\hline & Electricity cost & Per year $0.29 \times$ totalFlowRate \\
\hline & Battery depreciation cost & Per year1000 \\
\hline & Labor cost & Per year14.4 \\
\hline Total operating cost & & Peryear1449.4+0.29×totalFlowRate \\
\hline
\end{tabular}

The cost of a battery swap station includes initial investment cost and operating cost. The detail cost could be found in Table 2[9].

Sensitivity on TLR, battery, and construction cost. We compare ratio of EVs when TLR is 0.08,0.10 and 0.15 and construction cost, battery cost and monthly labor cost are set as 40 million, 40 million and 12 thousand Yuan. As we could see in Fig. 4, the higher technical learning rate is, the faster electric cars diffusion will be. Moreover, to technical learning rate 0.08 , the increase of 0.02 TLR can hardly bring improvement to EV diffusion. The uptake of EVs finally remain stable at 85\% after 10 years. Compared to fast charging stations with same TLR level, fast charging stations are more effective than battery swap stations on promoting the diffusion of electric cars. To keep the diffusion effectiveness in battery swap mode the same as fast charging mode, more money should be put to enhance the technical learning rate.

To see the influence of battery cost to the diffusion of electric cars, we compare the ratio of EVs, when battery cost is set in 20 , 30, 40 million Yuan, and TLR is set in 0.1,construction cost is 40 million Yuan, and monthly labor cost is 12 thousand Yuan. From Fig. 5, we could see that the lower battery cost is, the faster EV diffusion will be. Two curves representing for 30 and 40 million Yuan battery cost coincide, so when battery cost is 30 million Yuan, the electric diffusion is not sensitive to 10 million battery cost subsidy, or we could say when battery cost is 40 million Yuan, the electric diffusion is not sensitive to extra 10 million battery cost burden. According to our estimation, battery cost of each battery swap station is likely to be 50 million Yuan. 10 million cost subsidy could dramatically improve the effectiveness of electric cars diffusion from about 55\% to 95\%. However, another 10 more million subsidy won't bring any improvement. Therefore, we believe government should put 10 million subsidy as battery cost if they want to enhance uptake of electric cars.

Moreover, we compare ratio of electric cars when construction cost is 30, 40 and 50 million Yuan and TLR, battery cost and monthly labor cost are set as 0.1, 40 million Yuan and 12 thousand Yuan per month. As we can see in Fig. 6, the higher construction cost is, the slower electric cars diffusion will be. When construction cost is 50 million Yuan, 10 million Yuan construction cost subsidy will improve uptake of electric cars from 24\% to $60 \%$. Another 10 more million Yuan subsidy will bring another $20 \%$ improvement to $80 \%$. According to our estimation on construction cost, 50 million Yuan, 10 million subsidy is most effective. Moreover, compared to fast charging mode, battery swap mode is less effective to improve EV diffusion as it requires much higher cost.

Moreover, we also made simulation on labor cost influence to EC diffusion. The result shows labor cost has little influence to EV diffusion in battery swap mode, so we won't describe the detail here. 


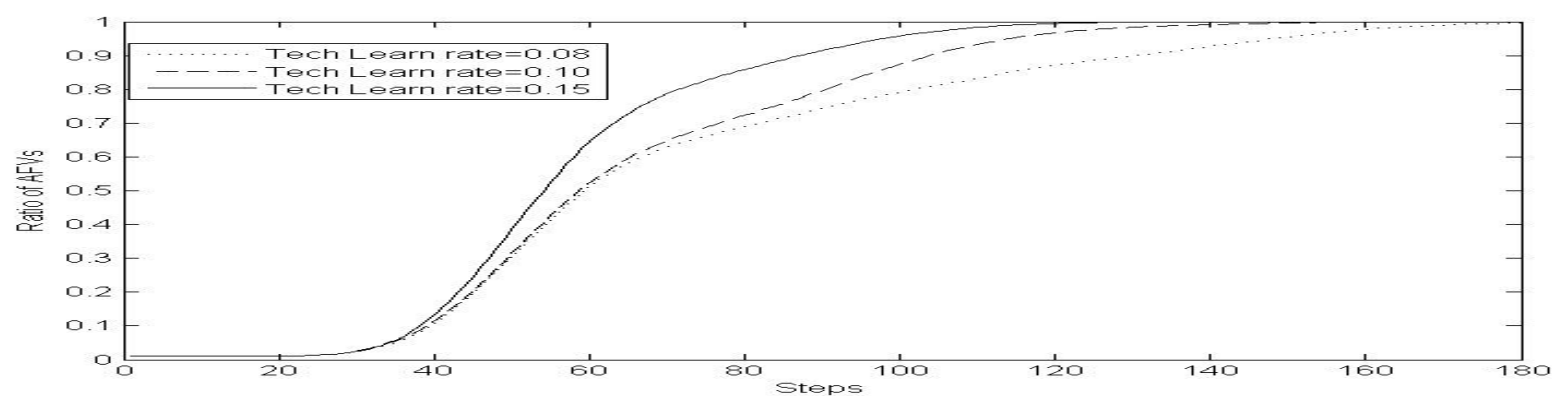

Fig.1. For FC, ratio of EV in different technical learning rate

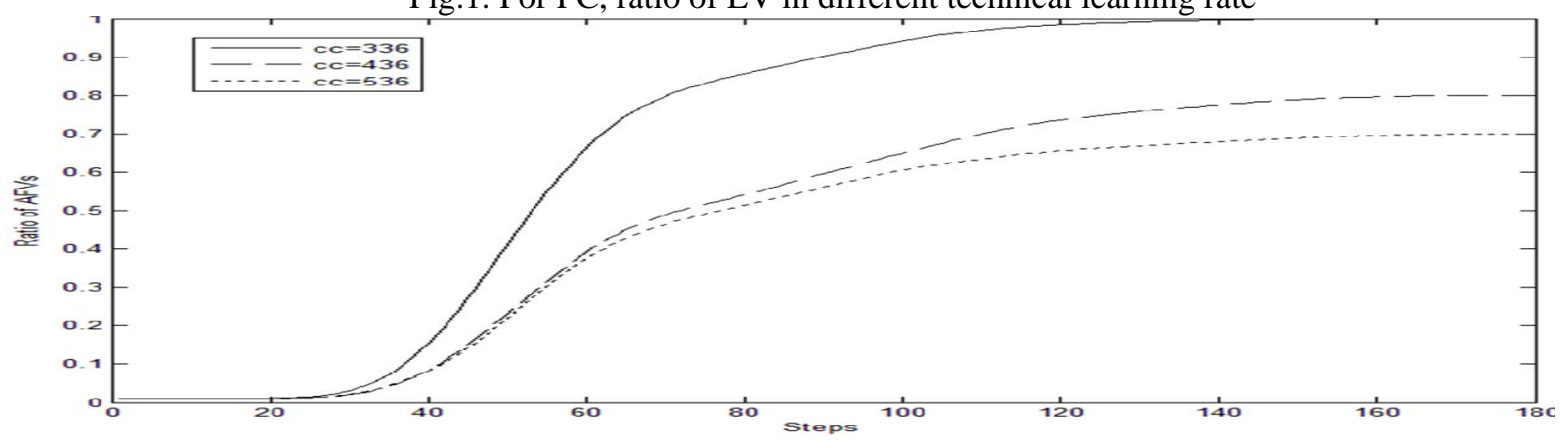

Fig.2. For FC, ratio of EV in different initial investment cost

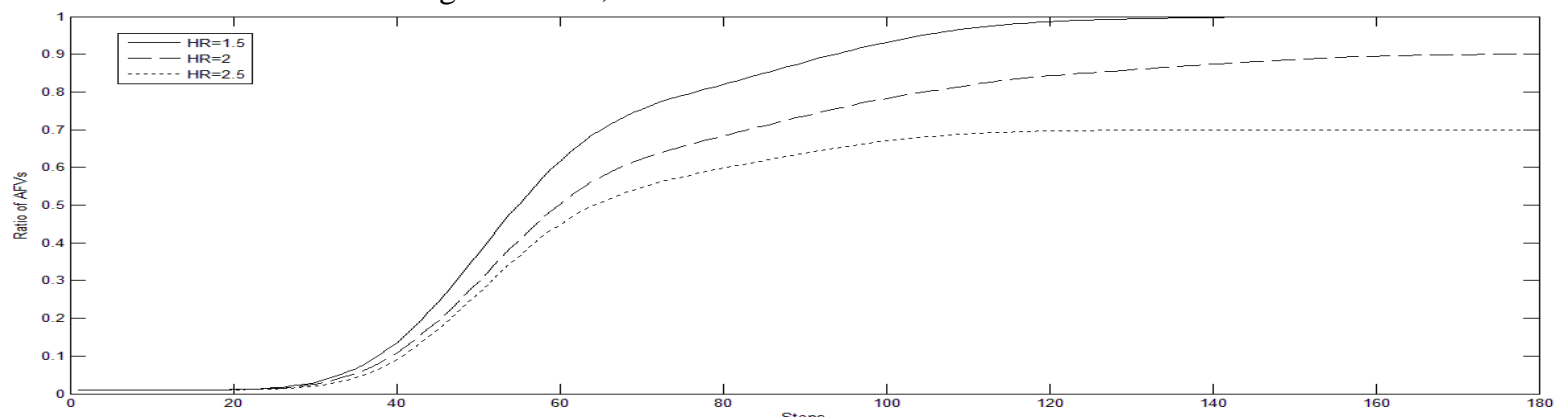

Fig.3. For FC, ratio of EV in different labor cost

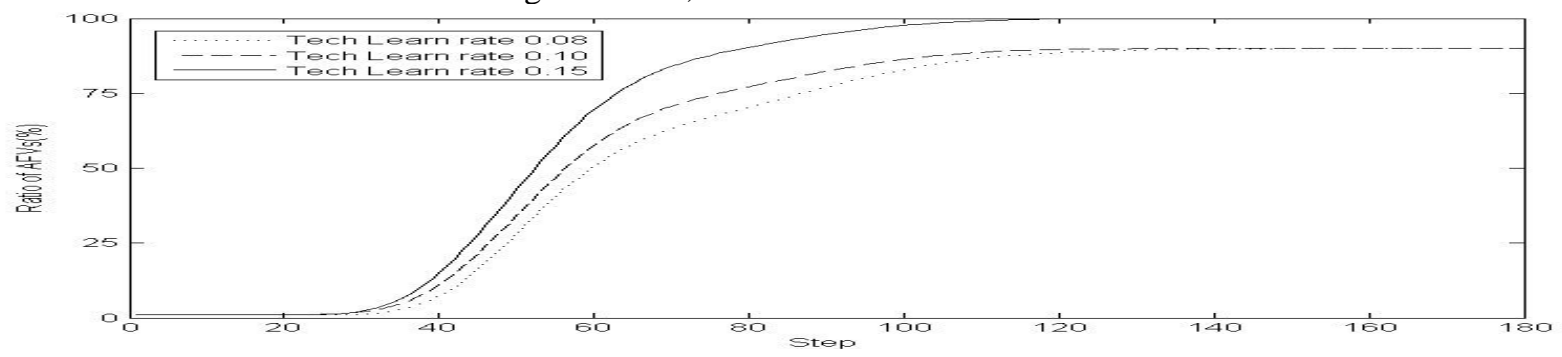

Fig.4. For BS, ratio of EV in different technical learning rate

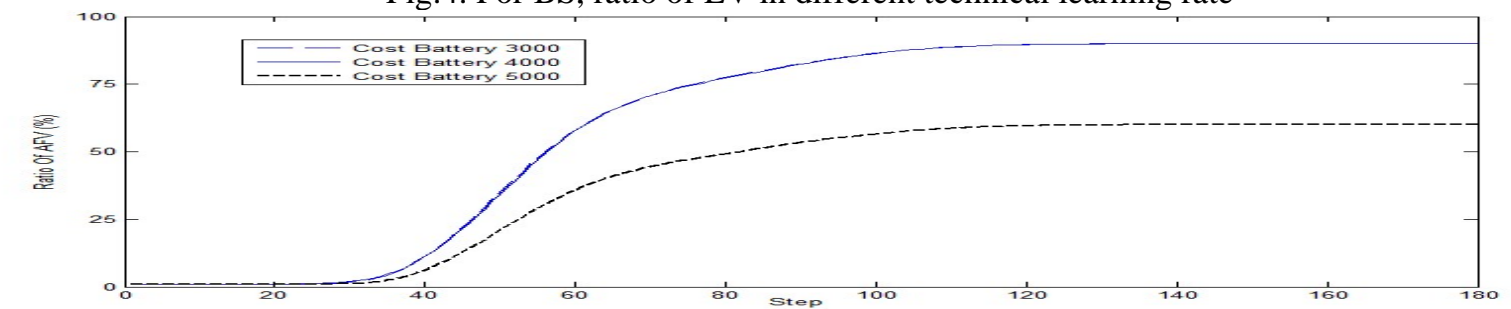

Fig.5. For BS, ratio of EV in different battery cost

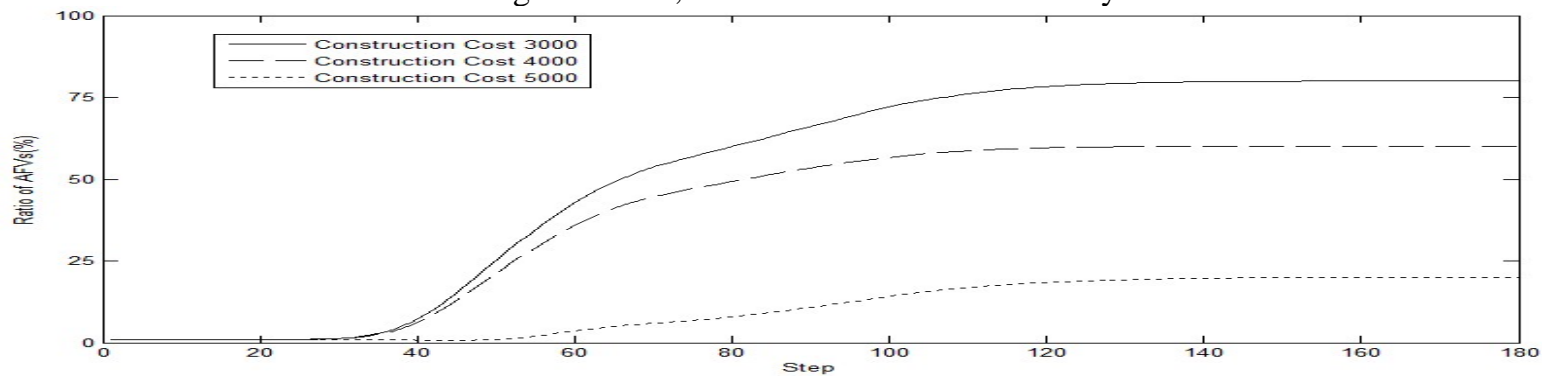

Fig.6. For BS, ratio of EV in different initial investment cost 


\section{Conclusions}

We find the higher TLR is, the faster and more effective the diffusion is. The lower cost is, the faster the diffusion is. For fast charging mode, initial investment cost have the highest influence on diffusion. For scenario where TLR is set in 0.1, construction cost, labor cost are set in 4.36 million and 20 thousand Yuan, EVs diffusion is more sensitive to construction cost decrease. For government who want to improve the uptake of EVs through subsidy on EV infrastructure of fast charging stations, we suggest they should put their subsidy on initial investment cost instead of daily operating cost. For battery swap mode, TLR, construction cost, and battery cost have influence on EV diffusion We have found scenario where TLR is 0.1 , construction cost 40 million, labor cost is 12 thousand Yuan, and battery cost is 40 million Yuan, electric cars diffusion is insensitive to 10 million Yuan battery cost decrease and sensitive to construction cost decrease. Therefore, we believe government should put subsidy on battery swap station's construction cost instead of battery cost. Moreover, to improve TLR, government should provide more preferential policy to nurture advanced technology to improve the electric cars' research and development ability.Comparing FC with BS on its effective influence on EV diffusion, we have found fast charging is more effective to improve diffusion speed and effect, as it enjoys lower cost. Therefore, we believe fast charging is more effective to improve EV diffusion, compared to battery swap.For future study, we will try to take time cost into consideration, as battery swap stations could finish refueling much faster than fast charging stations, which will attract consumers who are lack of time and don't want to wait.

\section{Acknowledgement}

This research is sponsored by National Nature Science Foundation of China (No. 71125002), and all shortcoming remains to the author.

\section{References}

[1] Jiancui L. Resource Science, 2011, 33(4)(In Chinese)

[2] Klabjan D, Sweda T. Wiley Encyclopedia of Operations Research and Management Science, 2011.

[3] Jiguang K, Zhenlin W, Danming C. Electric power demand management, 2009, 11(5): 64-66.(In Chinese)

[4] Tianchen H, Jingcai H. computer simulation, 2006, 22(9): 58-60.(In Chinese)

[5] Eppstein MJ, Grover DK, Marshall JS, et al. Energy Policy, 2011, 39(6): 3789-3802.

[6] Grau Unda I, Papadopoulos P, Skarvelis-Kazakos S, et al. Electric Power Systems Research, 2014, 110172-179.

[7] Shijian Xiang, Tieju Ma. Journal of management science, 2014, 17(1): 1-10.(In Chinese).

[8] Schroeder A, Traber T. Energy Policy, 2012, 43136-144.

[9] Wiederer A, Philip R. 2010. 